\title{
A User-Defined Web. And, on Systems modification in general.
}

\author{
Camille Akmut
}

\begin{abstract}
In no-television and permanent-vacation, we explore two programs that would fit into a wider User-Defined Web : a term chosen and adopted here in opposition to the however well-meant, but ultimately false, established concepts of a "user-centered" or "user-centric" Web - in so far as they are dependent on the good will of those who have control over the Web and Internet, their natures, developments and directions, in particular those who have an above-average influence on them. We discuss Systems modification generally : programs that change the way we think of technology and society, by often modifying both.
\end{abstract}


"the systems, both human and computer" (Berners-Lee) 


\section{Background : the Web and the Internet}

\subsection{The searches for reasons}

In other sciences and disciplines, a non-negligible part of the activity of scientists and scholars consists in and revolves around research seeking to establish the "etiology" of their various objects. Etiology, a term that, simply, means "root causes" or "reasons of being"; one that is strongly connected to the medical sciences. Why does a given thing exist? How did it come to be? Where and how did it first start? And, so forth. Such questions play an important role in the studies of infectious diseases for instance, as part of epidemiology. ${ }^{1}$

In some, the term etiology is replaced or is predated by others, with sometimes important inflections, and sometimes they are used or conceived of in direct opposition to it $^{2}$.

A major part of philosophy for instance is a subfield called "metaphysics" (a term that is conventionally motivated by the fact that it historically originated in the parts of Aristoteles' writings that dealt with this topic being placed after his book on Physics, hence literally meta-physics, or that which comes after, or beyond Physics), which can be described as the investigation of all questions that relate to the nature and purposes of our existence ${ }^{3}$.

For reasons that may be related to its relatively young age, and arguably its immaturity, in some ways, computer science and computer scientists have shown and display relative little interest in such endeavors and activities. Being still pioneers of their discipline, they may be more interested in doing, and being without having to worry about the more meta-theoretical questions that come, whether they wish it or not, with these same doings and beings, and their

\footnotetext{
${ }^{1}$ To give a specific example : the mortality rate among women giving birth, the etiology of which is now very different from how it was first conceived of during the 19th century : Semmelweis, the founder of modern antiseptic, or hygiene, rules, ultimately posited that much of it could be attributed to the practices of doctors at the time, some of whom went from dissecting corpses to assisting in birth, without disinfecting their hands and sometimes without changing their tools, practices that now appear as folly to us, but were not uncommon in the days of Semmelweis, who himself had struggled to come to this answer, both for scientific - the exclusion of competing hypotheses - and sociological reasons - this was not the standard of his time. Semmelweis, for his methods and hypotheses, though proven successful, was shunned and ostracized by the medical community, which in its wisdom held that, because things had been done a certain way, for a long time, there was no good reason to change (them). Most of the information presented here is derived from the medical doctoral thesis of Celine, of the same name, but the reader will most likely find it useful to consult additional sources.

${ }^{2} \mathrm{~A}$ controversy exists for instance as to whether etiologies of sexualities, of which many sexologists readily admit we know very little, should be taken into account when discussing and founding politics, human rights and legal acts that pertain or relate to them. Other controversies center around the notion of "essentialism" (when a mere property of a system is confused with or mistaken or passed for its nature).

3 The question "Why is there something rather than nothing?", posed by Leibniz at the beginning of the 18th century, is an often-quoted example of such questions; though Plato's inquiries into the "real nature of things", as perceived by our senses, and as posited as ideal, also represent a, perhaps more familiar, iteration of this particular sub-field's interests.
} 
meanings and functions including in our societies.

This may also be related to the context in which computer science was founded as a discipline, having had to deal with the skepticism of neighboring disciplines, and perhaps having adopted as an antidote a sort of hyper- but unrealistic scientism as a basic reaction.

Astronomy, a discipline not too distant, though much older, and within it in particular cosmology seems much more open to such questioning : it could have been limited to a simple cartography of the knowable universe, but instead questions surrounding the origins of the universe, its meanings, the philosophical implications of other life forms, are not foreign to them, and not treated as nonscientific, but in fact are treated as questions of first-rate scientific importance, and anyone able to answer them would forever have a place in history. This attitude seems to extend to some parts of physics as well.

In the same sciences and disciplines described above, whether misguided or not, inquiries into "the origins" or reasons of being are (can be) used as some indicator or guide of "this is what this truly is or means"; or with the political or philosophical or even aesthetical implications of "this is the purest form, or this is what we ought to want or get back".

In the historical sciences, in particular as conceived and practiced by the Annales school $^{4}$, research into previous eras can serve as a mirror to our own times; it is thus endowed with some subversive quality, if not political potential which can be summed up as : "if this was so, then, then what is now is only one possible, and hence transformable version of our daily experiences; and, equally, whatever we have gained is something we can lose again".

But, when reaching the question "Why the Internet?", one is dumbfounded and hard pressed to find many answers in computer science literature. The obvious approach consisting in looking at writings within networking or networks, the particular sub-field of computer science dedicated specifically to these topics, yields relatively little results.

These authors generally prefer to concentrate on the "purely technical" aspects (of their discipline), under the silent though widely accepted notion of there being such a thing ${ }^{5}$.

\footnotetext{
${ }^{4}$ Annales is a journal around which gravitated a series of important figures within history that have (since) then been subsumed under that banner : Marc Bloch and Lucien Febvre in particular, and others. They all had a certain sensibility for, and art in approaching topics that, while at first sight remote, both in time and nature, from our own current concerns, nonetheless offered potent insights into our lives (e.g. Lucien Febvre's research into the powers attributed to rulers, both given and acknowledged).

${ }^{5}$ To such approaches, one could oppose a diametrically opposite one, that in computer science everything is political, from the first chip, down to the most abstract algorithm. A notion no doubt highly irritable to these purists - though only time will tell if theirs can hold much longer.
} 


\subsection{The Internet, and its past}

Andrew Tanenbaum starts his much used textbook ${ }^{6}$ with an introduction that gives an overview of the subject and ends with an extensive bibliography.

Conforming with the tradition established by such books, he presents the topics of networks according to a layer approach : layer 1 (the physical layer), layer 2 (the data layer), etc, etc.. Except for the strange choice of explicitly opting for "he" to mean "he or she", there is nothing exceptional about this book or its approach ${ }^{78}$.

Adopting the "top-down" approach for which they are known ${ }^{9}$, which simply reverses the layer approach but does not alter it, Kurose and Ross choose to go from the surface and the most familiar, i.e. by starting with what appears on the screen for instance, the web pages in other words, down to the less visible or approachable, the various data layers and packets; an approach that simply chooses to go backwards the already established model as we have already stated $^{10}$.

All such presentations, whether "top-down", or bottom-up as in the case of Tanenbaum ${ }^{11}$, more or less follow one or the other of the variants of the OSI (layer) model, also sometimes found as ISO, or a comparable one's variants (TCP/IP).

Much is found about the what's and how's while much remains unclear about the why's of it all. The authors may be of the opinion that such questions have no place in a scientific or college publication; or that they are the domain of other scientists.

Such books, primarily directed at college audiences, are however not completely devoid of interest when trying to find some historical, if not philosophical reasons for the existence of the Internet or Web. In Tanenbaum ${ }^{12}$, we learn crucially that ${ }^{13}$

Traditionally (meaning 1970 to about 1990), the Internet and its predecessors had four main applications:

\footnotetext{
${ }^{6}$ Computer Networks. We use the last edition published by Tanenbaum alone, the fourth edition (Tanenbaum 2003).

${ }^{7}$ As we explain below. (Elsewhere, however, we highlight specific weaknesses and give a more detailed critic of this book's more recent successor in particular.)

${ }^{8}$ It would appear more wise to use they, as in "they have to log on that machine and run it there", if a gender-neutral or gender-all-encompassing approach was sought.

${ }^{9}$ Kurose and Ross 2017.

${ }^{10}$ Accordingly, they start with the so-called Application Layer, which contains the Web and its various protocols (HTTP), in chapter 2. Ibid.

11 "He starts with an explanation of the physical layer of networking, computer hardware and transmission systems; then works his way up to (...)" (Description for Tanenbaum 2003).

${ }^{12}$ Tanenbaum 2003. Chapter 1. Specifically : "1.5 Example Networks", which includes the Internet, ARPANET, NSFNET, etc., and historical developments on each.

${ }^{13}$ The more or less the same presentation, but in less detail, is found in Kurose and Ross 2017 on page 126 .
} 
1. E-mail. [since ... ARPANET] ...

2. News. [Newsgroups] ...

3. Remote login. [telnet, rlogin, or ssh] ...

4. File transfer. $[\mathrm{FTP}] \ldots$

Up until the early 1990s, the Internet was largely populated by academic, government, and industrial researchers. One new application, the WWW (World Wide Web) changed all that and brought millions of new, nonacademic users to the net. This application, invented by CERN physicist Tim Berners-Lee, did not change any of the underlying facilities but made them easier to use. Together with the Mosaic browser, written by Marc Andreessen at the National Center for Supercomputer Applications in Urbana, Illinois, the WWW made it possible for a site to set up a number of pages of information containing text, pictures, sound, and even video, with embedded links to other pages.

Histories of the Internet, and the Web, have been written, and these offer more insights, or more detailed ones ${ }^{14}$.

But, before we turn to them, now is a good time to establish, and make explicit, some of the terminology that we use. A distinction has been made between the "Internet" and the "Web", and it would be purposeful to remind of what these differences are.

To quote from Tanenbaum, "the Internet is not a single network but a network of networks", while "the Web is a distributed system that runs on top of the Internet." 15

It also follows out of previous developments that : 1 . The Internet predates the World Wide Web (or just "Web"), and 2. The Web is a portion or a branch $^{16}$, in some presentations, of the Internet, or a structure that sits on top of it $^{17}$, in others. Out of these presentations, it emerges that the Web uses the Internet as some sort of medium, or perhaps conduit - to use flawed visual imagery.

Among such histories, Where Wizards Stay Up Late: The Origins of the Internet plays a particular role as one of the first books published on the topic

\footnotetext{
${ }^{14}$ But only so, in a system, where computer science was able to be successfully established as a discipline where most social concerns and political ramifications have been (successfully) removed or erased, a fact held by some as a definitive proof of its maturity, and by others as one of its great weaknesses and lacks.

${ }^{15}$ Tanenbaum 2003. Chapter 1.

${ }^{16}$ Hafner and Lyon 1998.

${ }^{17}$ Tanenbaum 2003.
} 
with a wide readership ${ }^{18}$, preceding both Abbate's treatment of the subject and Tim Berners-Lee's own, and presumably surpassing both in sales by a large margin. ${ }^{19}$

Here, journalist Katie Hafner and Matthew Lyon tell a story of an Internet that had begun at the ARPA, Advanced Research Projects Agency, a part of the Defense Department, after which the eponymous ARPANET is named, that was able to strive in the context of an administration and specifically a President, Dwight Eisenhower, that had much faith in scientists and favored their company, and the Cold War (the launch of Sputnik had given a specific scientific twist to these wider oppositions $)^{20}{ }^{21}$

It is hard to read some of these beginning developments and not think of how much they appear in contrast with present times.

Eisenhower hadn't wanted a seasoned military expert heading the Pentagon; he was one himself. The president distrusted the militaryindustrial complex and the fiefdoms of the armed services. His attitude toward them sometimes bordered on contempt.

By contrast, he loved the scientific community. He found scientists inspiring-their ideas, their culture, their values, and their value to the country-and he surrounded himself with the nation's best scientific minds. ${ }^{22}$

Of the period mentioned by Tanenbaum that preceded the Web, where one of the main applications was e-mailing, not browsing, Hafner and Lyon write :

In the decade before the ARPANET, computer scientists had devised ways of exchanging electronic messages within a time-sharing system. Researchers on the same time-sharing system each had a designated file, like an in-box, in the central machine. Colleagues could address short electronic messages to someone else's box, where

\footnotetext{
${ }^{18}$ Where Wizards Stay Up Late was - as the cover of the 1998 paperback edition indicates - a "National Bestseller".

${ }^{19}$ Where Wizards Stay Up Late was published for the first time in 1996, in a hardcover edition by Simon \& Schuster, then again in 1998 in paperback form, Abbate's Inventing the Internet in 1999, Berners-Lee's Weaving the Web in 1999 also (at which point world usage of the Internet was measured to be at about $5 \%$ of the world population compared with $50 \%$ currently, a large part of it driven by the Web).

${ }^{20}$ ARPA, now DARPA (Defense ...), was founded by Eisenhower in February 1958. In response to the launch of Sputnik (October 1957).

${ }^{21}$ The exposition in Where Wizards Stay Up Late is and reads much like a novel or a long work of investigative journalism : their story starts for instance with a flashforward to 1994. It lacks indication of sources within the text, e.g. in the form of notes, but features an extensive bibliography at the end (where the reader is left with the unpleasant task of putting the pieces together). Their non-descriptive choices of titles for the chapters of their book make it hard to follow them.

${ }^{22}$ Hafner and Lyon 1998. Chapter 1.
} 
only the recipient could read them. Messages could be dropped and picked up at any time. It was convenient, given the odd hours people kept. People within a single lab sent parades of one-liners back and forth, as well as longer memoranda and drafts of papers.

The first of these programs, called MAILBOX, was installed in the early 1960s on the Compatible Time-Sharing System at MIT. Similar mailboxes became a standard feature of almost every timesharing system built thereafter. ${ }^{23}$

And, later, on the same topic, and the same chapter, we find that

In 1973, Lukasik ${ }^{24}$ commissioned an ARPA study that found that three quarters of all traffic on the ARPANET was e-mail.

First mentions of the ARPANET appear in chapter 6, while first mentions of the ARPA network start appearing at the end of chapter 2 .

But, other networks, whose purpose was primarily to link scientific communities together, are evoked, in particular the NSF's CSNET (a network to connect members of the computer science community) and the later NSFNET that catered to a wider public of scientists ${ }^{25}$.

Linking the computer science departments to the ARPANET was out of the question. To be assigned a site, universities had to be involved in specific kinds of government-funded research, typically defense-related. Even then, it was costly to allocate new sites. ARPANET connections came in one size only: extra large. The system used costly leased telephone lines, and each node had to maintain two or more links to other sites. As a result, maintaining an ARPANET site cost more than $\$ 100,000$ each year, regardless of the traffic it generated.

The computer scientists had to invent another way. In May 1979, Larry Landweber, head of the computer science department at the University of Wisconsin, invited representatives of six universities to Madison to discuss the possibility of building a new Computer Science Research Network, to be called CSNET. ${ }^{26}$

The book closes on an epilogue, where the Web appears, Tim Berners-Lee, his HTTP protocol, and the CERN code library are mentioned.

\footnotetext{
${ }^{23}$ Hafner and Lyon 1998. Chapter 7 "E-Mail".

${ }^{24}$ Lukasik is Stephen Lukasik, the seventh director of (D)ARPA. See, https://www.darpa. mil/attachments/DARPA_Directors_Sheet-web.pdf

${ }^{25}$ Hafner and Lyon 1998. Chapter 8.

${ }^{26}$ Ibid.
} 


\subsection{The Web, and its future}

Still, histories of the Internet, and here specifically the Web, have been written or produced by some of their creators.

One important such book is Tim Berner's Lee Weaving the Web: The Original Design and Ultimate Destiny of the World Wide Web ${ }^{27}$.

The tone and approach of Weaving the Web is very different from both the textbooks and historical accounts cited previously, not least attributable to the unique position of its author as inventor of many of the technologies underlying and of the Web.

When I first began tinkering with a software program that eventually gave rise to the idea of the World Wide Web, I named it Enquire, short for Enquire Within upon Everything, a musty old book of Victorian advice I noticed as a child in my parents' house outside London. With its title suggestive of magic, the book served as a portal to a world of information, everything from how to remove clothing stains to tips on investing money. Not a perfect analogy for the Web, but a primitive starting point. What that first bit of Enquire code led me to was something much larger, a vision encompassing the decentralized, organic growth of ideas, technology, and society. The vision I have for the Web is about anything being potentially connected with anything. It is a vision that provides us with new freedom, and allows us to grow faster than we ever could ${ }^{28}$

And, for the first time, we also find expressed, in no unclear terms, with no ambiguity, an answer to our original inquiry : "Why a Web, why the Web?"

\section{a vision encompassing the decentralized, organic growth of ideas, technology, and society. ${ }^{29}$}

It is worth noting that the sources Berners-Lee draws from throughout the book, from literature to computer science, are also in stark contrast with preceding treatments, painting the picture of a scientist and technologist with broad interests.

These philosophical and literary underpinnings are usually then found erased, or purified, in other publications ${ }^{30}$.

\footnotetext{
${ }^{27}$ The first, 1999 edition still featured the full title Weaving the Web: The Original Design and Ultimate Destiny of the World Wide Web by Its Inventor.

${ }^{28}$ Berners-Lee 1999 : 1.

${ }^{29}$ Ibid.

${ }^{30}$ As to why, we have already suggested so : the cult of the "purely technical" among many computer scientists. These computer scientists, put shackles on each other, and shackles on others, but many of them see no obvious complications or moral dilemmas on the other hand in accepting morally-ambiguous positions at various, but always similarly dubious and morally-ambiguous companies of morally-uncertain objectives, characters and/or acts.
} 
The story of how the Web was created has been told in various books and magazines. Many accounts I've read have been distorted or just plain wrong. The Web resulted from many influences on my mind, half-formed thoughts, disparate conversations, and seemingly disconnected experiments. I pieced it together as I pursued my regular work and personal life. I articulated the vision, wrote the first Web programs, and came up with the now pervasive acronyms URL (then UDI), HTTP, HTML, and, of course, World Wide Web. ${ }^{31}$

Berners-Lee does not try to embellish the story. The Web, when it was first conceived, was far from an immediate success, including failings in securing funding or convincing colleagues. The modest origins of the Web are rendered in passages such as :

Robert $^{32}$ and I wrote a paper, too, "Hypertext at CERN," which tried to demonstrate the importance of what we were doing. What we hoped for was that someone would say, "Wow! ..." But it didn't happen.

In June we held talks and demonstrations within CERN, and wrote about the Web in the CERN newsletter. ${ }^{33}$

And, though, the Web appears now to us as a vast, anonymous, directionless space, this book helps remind that the Web, like most of the rest of technology (we use), is the creation of people, who watched and observed with some worry from afar, some more active or proactive than others, and that the Web did grow from something small (one server(?)) into what we know today :

As the Web slowly spread around the world, I started to be concerned that people who were putting up servers would not use HTTP, HTML, and URIs in a consistent way. If they didn't, they might unintentionally introduced roadblocks that would render links impotent.

$(\ldots)$

By now the Web consisted of a small number of servers, with info.cern.ch the most interconnected with the rest. ${ }^{34}$

\footnotetext{
${ }^{31}$ Ibid. : 2.

${ }^{32}$ Robert is Robert Cailleau, of whom Berners-Lee writes earlier in the book : "While it seemed to be uphill work convincing anyone at CERN that global hypertext was exciting, one person was an immediate convert: Robert Cailliau." (Ibid. : 25).

${ }^{33}$ Ibid. : 46.

${ }^{34}$ Ibid. : $53 ; 55$.
} 
If the criterion of "What should the Web be?" is made to be what its creators intended it to be, then we must hold that the Web was envisioned as a network of communication of information, with some emphasis placed, historical if not philosophical, on knowledge (with some scientific standard attached to it).

This seems at first sight preferable to a Web dominated by commercial interests and advertisements - though only in a society where knowledge was held to be of higher value than financial pursuits.

In his 30th open letter, from 2019, each marking one anniversary of the Web, Berners-Lee wrote :

We need open web champions (...) who will take action when private sector interests threaten the public good and who will stand up to protect the open web.

Companies must do more to ensure their pursuit of short-term profit is not at the expense of human rights, democracy, scientific fact or public safety. ${ }^{35}$

As for the Internet, many of the early networks, defense applications that have almost always accompanied the development of computer science excluded, were also thought of as ways to link creators and consumers of knowledge together (through e-mail).

Critics of such a position will argue that, due to their free and dynamic nature, the Web and the Internet are whatever they are at any given time, and that they should not be made to be anything specific - a position that can be described as what it is here, namely, a laissez-faire position of the category "this is just how things are, this is how they've always been", and one that objectively supports the current status quo, which is highly in favor of large companies over users.

\section{User-Defined Web}

\subsection{Dominant language : "user-centered", "user-centric"}

"The ideas of the ruling class are in every epoch the ruling ideas, (...) The class which has the means of material production at its disposal, has control at the same time over the means of mental production, so that thereby, generally speaking, the ideas of those who lack the means of mental production are subject to it." posited Marx.

Much of our language defines our thoughts, and when using established terms such as "user-centered" or "user-centric" Web, we use terms, presumably having

\footnotetext{
${ }^{35}$ Berners-Lee 2019.
} 
originated in or emerged out of user interface theory or practice, academic or corporate, though this would be the subject of an entirely separate research of its own, that have become dominant, and they have so because they are regularly used by large companies, and so only as they serve their interests ${ }^{36}$.

\section{Autodesk}

June (...) 2018

User-centered design starts with empathy. Design leader (...) talks innovation and making a difference worldwide.

https://www.autodesk.com/...

\section{Measuring the User Experience on a Large Scale: User- Centered Metrics for Web Applications}

Kerry Rodden, Hilary Hutchinson, and Xin Fu

Google

There is a strong need for user-centered metrics for web applications

In this note, we describe the HEART framework for user-centered metrics, as well as a process for mapping product goals to metrics. We include practical examples of how HEART metrics have helped product teams make decisions that are both data-driven and usercentered. ${ }^{37}$

Falsely benevolent, to not so say patriarchal, in so far as they have the false property of not only being the product of top-down, few-in-the-know-many-inthe-dark models of creations, no matter how many samples they may collect, but also to depend on the good of will of these few, select companies - whose verifiable past practices ${ }^{38}$ (be it their handling of private user data, their affinity with tax avoidance, among many other things noted in many other places) by almost all thinkable metrics leave much to be desired.

These terms themselves most likely go back to the established user-centered design (UCD) or user-driven development (UDD) out of design theory ${ }^{39}$.

No matter what endearing terms they are attached - here "HEART" - they rely on the old models of a Web by few for many. A version of the Web whose failings keep manifesting. Models that are those of traditional media, television first. Failings that the creator of the Web, Berners-Lee, himself highlights.

\footnotetext{
${ }^{36}$ It cannot be reasonably assumed that they were chosen or picked because they did not, as otherwise terms such as "user-annihilating", which may in other ways arguably better, objectively describe their practices, would be dominant instead. They are not for obvious reasons.

${ }^{37}$ Rodden at al. 2010.

${ }^{38}$ As opposed to promises, of wanting to "do better".

${ }^{39}$ And, The Design of Everyday Things.
} 


\subsection{Definition : User-Defined Web}

Instead, we choose the term User-Defined Web that transparently translates into : a Web defined by its users (where each user, as in theory in a democracy, has the same weight). And, specifically not : a Web defined such that it would be thought to be agreeable to users (by few taste makers ${ }^{40}$ ). ${ }^{41}$

\subsection{Internet consolidation, and the Web of Companies}

This is not the case at the moment. Large corporations have an overwhelming influence on the Web, at present time. This, we can ascertain from various metrics on domain name popularity (e.g. https://www.alexa.com/siteinfo) and distribution of peak hour traffic ${ }^{42}$.

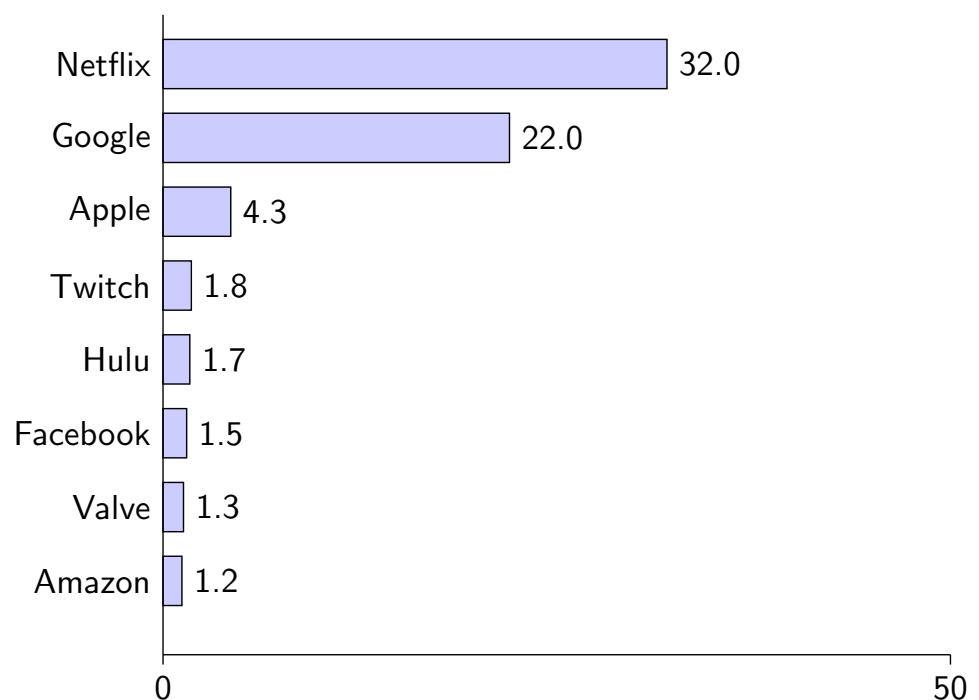

Percentage of U.S. peak Internet traffic produced by companies' networks, Feb. 2014 (DeepField/WSJ)

These eight companies alone were responsible for over $65 \%$ of total U.S. peak Internet traffic - as measured during the last week of January leading to February $2014^{43}$.

Two companies - Netflix and Google - subsumed among themselves $55 \%$ of all Internet peak traffic in a given week of 2014.

\footnotetext{
${ }^{40}$ E.g. Rodden et al., corporate designers (including company-hailed "design leaders", called so not without good reason), etc.

${ }^{41}$ A possible alternative for terminology could have been "user-driven", but this could be misconstrued to mean the exact opposite. Additionally, this terminology has already been charged with much history and practices, some of them implying very different things, including its inverse, as part of so-called "user-driven development" (a philosophy of design).

${ }^{42}$ The following chart is based on The Wall Street Journal, "Apple Quietly Builds New Networks". See FitzGerald and Wakabayashi 2014.

${ }^{43}$ A note attached to their graphic mentions "For week ending Feb. 3. Source: DeepField" (where DeepField is "network researcher DeepField Inc.").
} 
If we account for the further concentration that has taken place since then, Amazon having acquired Twitch, six months after the above statistics were measured, we get the following chart - a proxy only - for August 2014 :

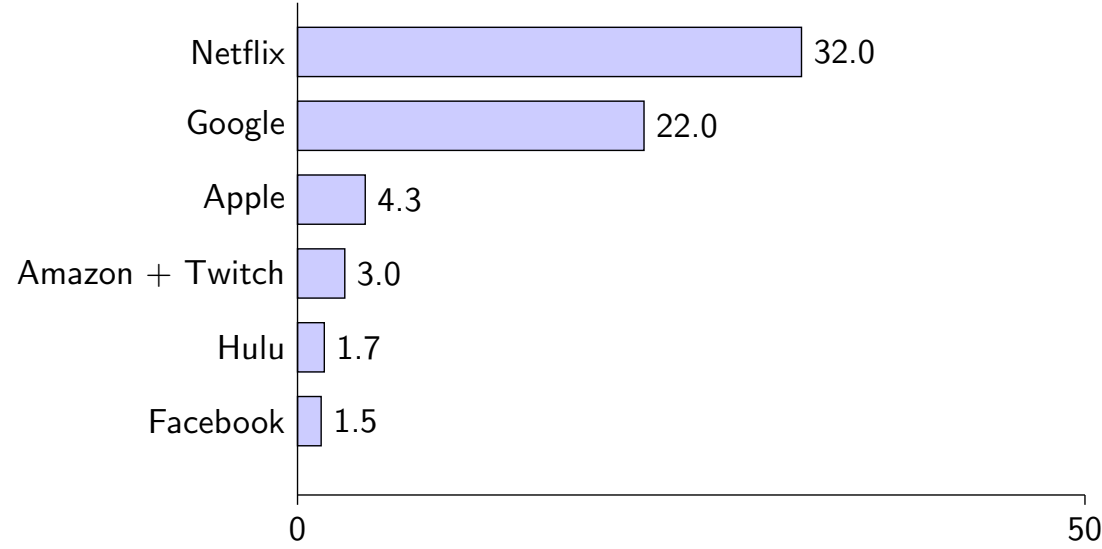

Percentage of U.S. peak Internet traffic produced by companies' networks, proxy Aug. 2014

Here, six companies alone - Netflix, Google, Apple, Amazon (incl. Twitch), Hulu (Disney majority-owned), and Facebook - are responsible for what can be approximated to be $65 \%$ to $75 \%$ of total U.S. peak Internet traffic ${ }^{44}$.

In his 29th open letter to the Internet, published in 2018, Tim Berners-Lee wrote :

What was once a rich selection of blogs and websites has been compressed under the powerful weight of a few dominant platforms. ${ }^{45}$

One particular striking example of the ways in which the Web has been successfully re-shaped by large companies is given by the website YouTube ${ }^{46}$, (currently) owned by Google.

In 2005, the year of its founding, Internet users would have found this website in the approximate following state during the month of December ${ }^{47}$ :

\footnotetext{
${ }^{44}$ In a follow-up article from May of that year, "Netflix's Share of Internet Traffic Grows", by the same publication and the same author, but drawing on different sources, Sandvine Corp., Netflix traffic was estimated to have grown to 34\% (FitzGerald 2014). We base our estimations on these trends having kept up.

${ }^{45}$ Berners-Lee 2018.

${ }^{46} \mathrm{https}$ ://www. youtube.com/

${ }^{47}$ The graphic we present is from a website called First Versions. The specific location of the image is https://www.firstversions.com/2016/01/youtube.html where it appears with the caption "YouTube, homepage at its official launch (December 15, 2005)". This state is also captured, and archived, as part of the Internet Archive here : https://web.archive.org/ web/20051215194008/http://www. youtube.com/ (December 15, 2005 19:40:08). We include this version in the appendix; to its significance, it can be added that it is the last available capture for that day.
} 


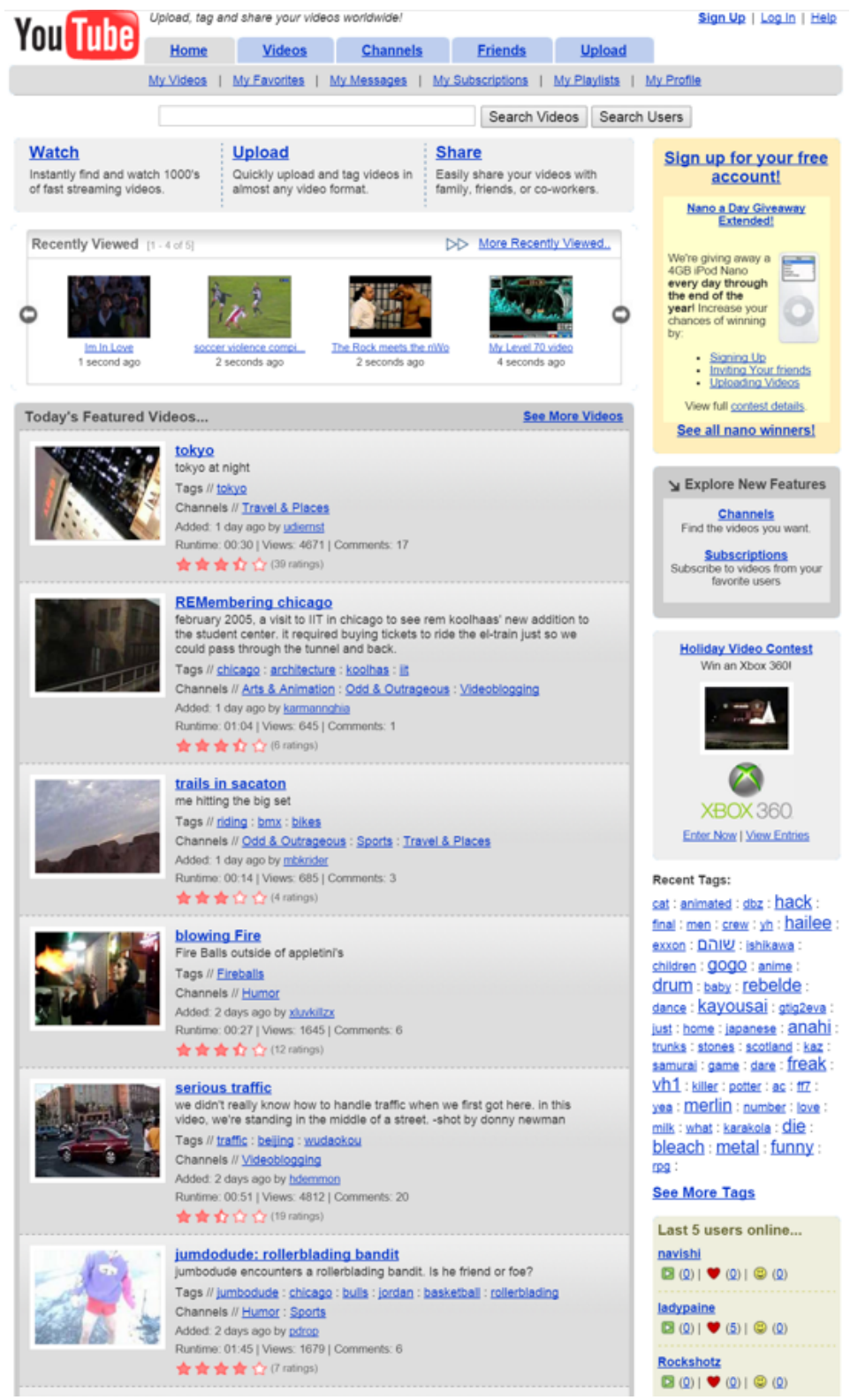

Figure 1: YouTube in 2005 ("official launch (December 15, 2005)").

Note, in particular, the large absence of videos by record labels, or film studios, or celebrities (including the particular strand of YouTube celebrities), that have now become common place on that same platform; having meanwhile undergone itself a change of ownership, from its original three founders, to one of the largest technology companies of our times.

It could be said, that these transformations have made websites like YouTube, and others like it, closer to the experience of comparable traditional media and 
in particular television, where much if not all of content is non-modifiable and corresponds largely to mainstream interests and follows corporate, for-profit dictates.

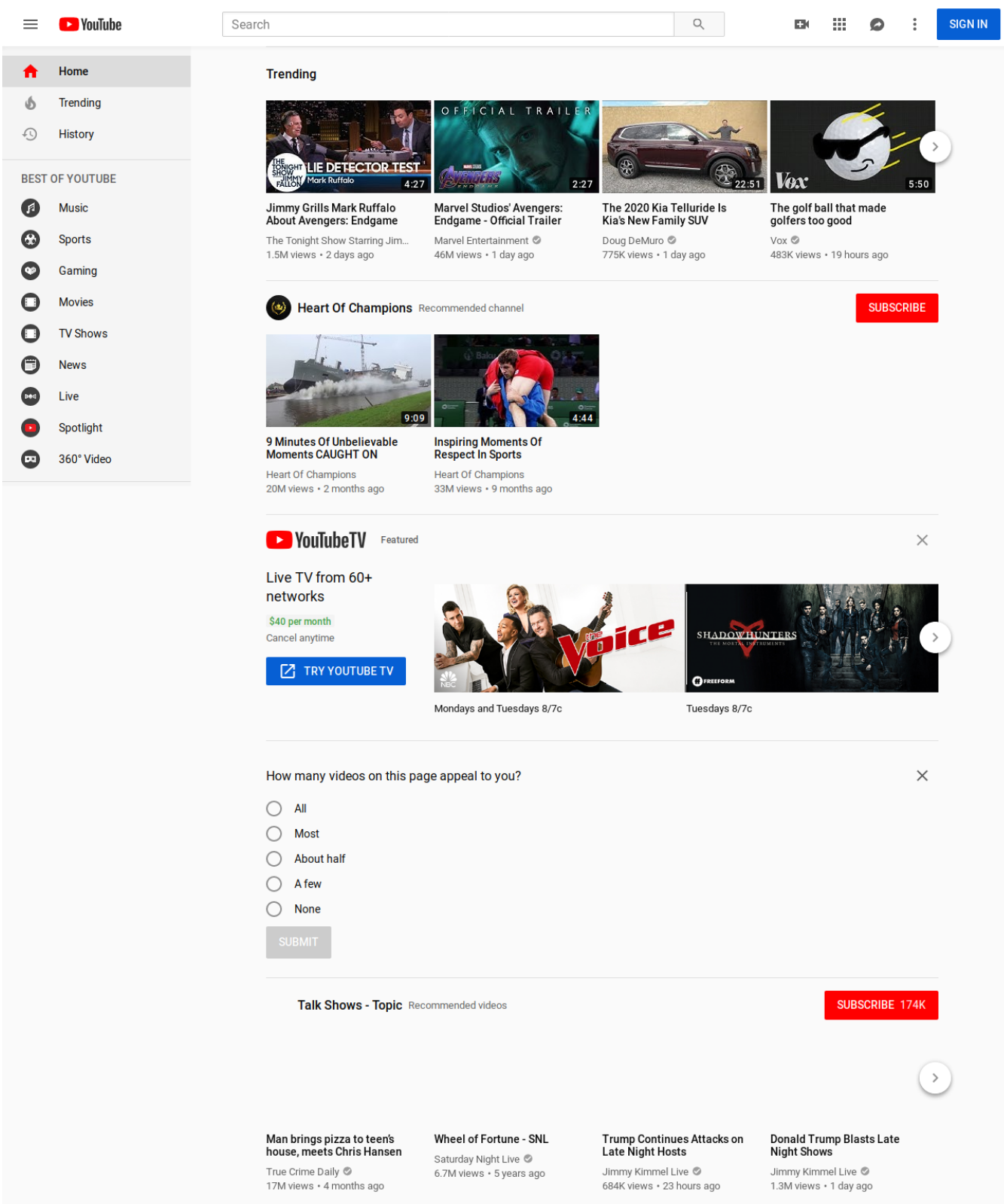

Figure 2: YouTube at present time.

Further, this occurs : 
With much of the same effects. It puts the viewer into a passive position of consumer of mostly unsolicited content rather than a free, independent, active explorer or shaper of information.

It is however not the absence of technical means that prevent us from creating alternatives.

\section{4 'no-television' and 'permanent-vacation', user-defined web programs}

In 'no-television' (appendix I) and 'permanent-vacation' (appendix II), we propose two programs that together offer a radically different view of what the Internet, Web could be : namely one where users each individually shape or rather re-shape the Web to their own liking, and preferences.

This goes beyond the mere selection of websites they visit, and the frequency at which they do so. But is extended to how it is rendered or presented to them; and, their ability to select - other than by looking or looking away what content they which to receive on these websites - independently from the wishes, objectives or intentions of their makers.

In 'permanent-vacation', as well as 'no-television' we explored, starting from a simple personal wish or need, attempts to shape and re-shape the contents, all of it done with only the most basic of programming.

The title of the first was chosen in reference to the eponymous film by Jim Jarmusch, in which the lines between reality and fiction are blurred; such that it is unclear whether this represents a dream-like version of ours, or a political alternative to ours ${ }^{48}$.

\footnotetext{
${ }^{48}$ In Permanent Vacation, his student film, most notably, work is largely or completely absent, among other common, widely-held for essential or indispensable parts of our lives (an interpretation that would have the merit of being a reasonable explanation for the title). Marked by empty streets, apartments empty of furnishings, absence of televisions, empty cells, etc. This program, as well as the other, no-television, also received much influence from the contents, mood and visuals of Stranger than Paradise, his follow-up film (motel, near airport, etc.). (This theme is also present in his later work.)
} 


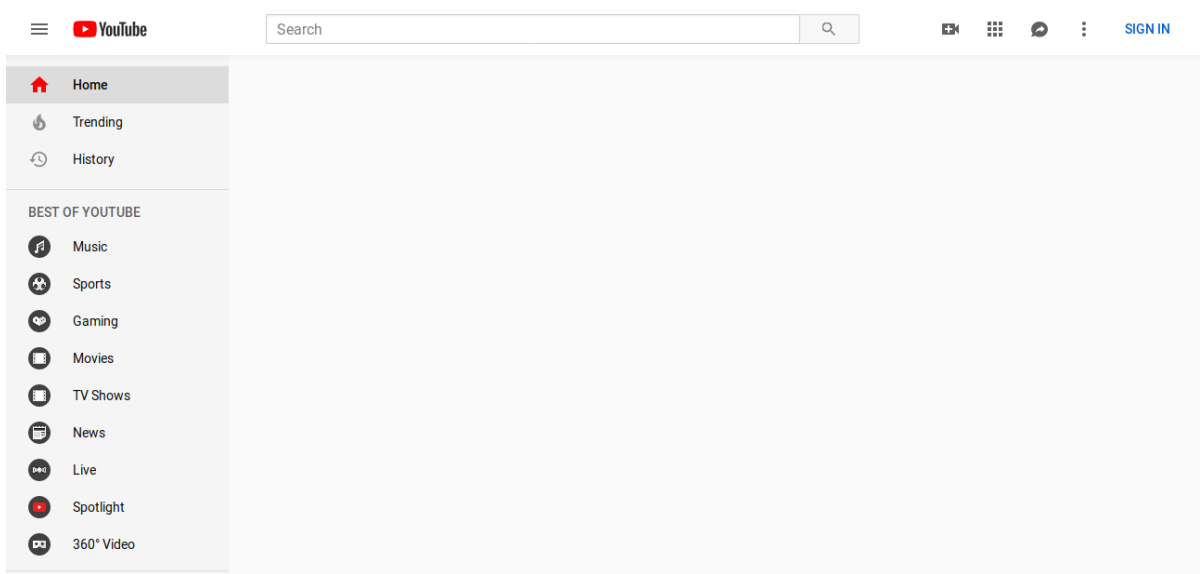

Figure 3: A user is greeted with the above interface while using no-television

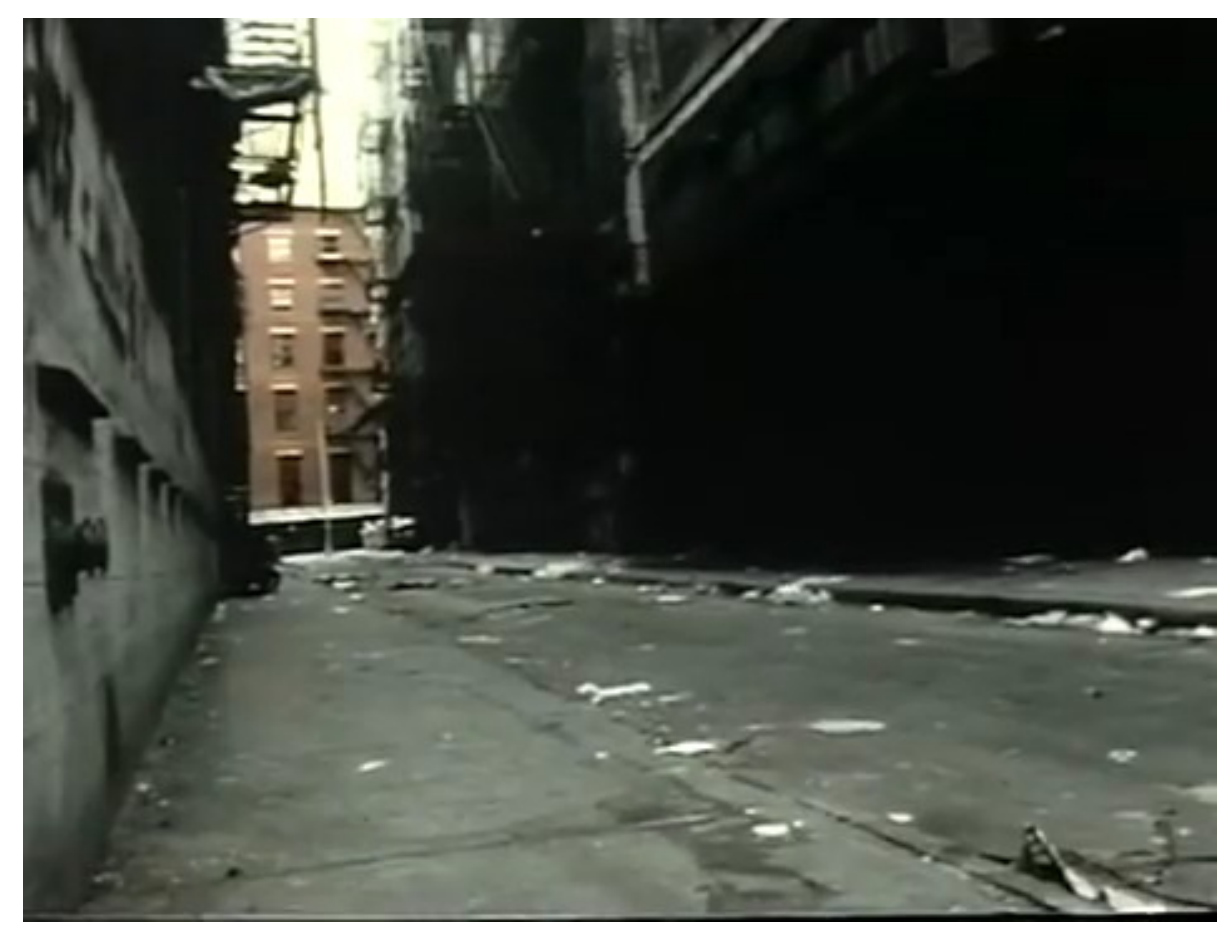

Figure 4: A scene from Permanent Vacation by Jim Jarmusch.

Though, we have described them as experimental, such programs are not uncommon and fit into the category of Browser plug-ins.

Indeed, such programs already exist, and some of them are in in fact in wide use. A category of tools called "ad blockers" serve as such an example : but, while their aim is to remove elements, namely advertisements, these are already commonly seen - hence also their usage - as something inherently foreign (of 
the Web) or distractive.

A more pernicious form of advertisement comes in the form of content passed as content, but whose nature is in fact identical or similar to that of an advertisement. Other contents, while not so, can nonetheless be seen as intrusive or unwanted, and users will want to remove them (certain contents or parts of a website that is of no interest to them, or has negative value to them).

But, there can be no universal programs for such use cases as we explain.

For these kinds of needs, there can be no widely used programs, as each user would most like differ in what they consider to be wanted and unwanted, including advertisements-passed-as-content; hence each user's needs would be different from the next.

(Other similar such programs, of smaller user numbers, target Javascript and do the same for Javascript by blocking it or its delivery according to a series of rules : but, here too, this targets elements of the Web that are seen as inherently exterior to it, as some (technical) nuisance or danger; the television equivalent, of not ads, but technical limitations imposed on the user by cable companies' equipment or so.)

Ideally, a Web could exist where each user defines, writes their own plug-ins so that they create their own version of the Web, that is not that of other users, and is not that envisioned by corporations either.

In other ways, It would make it so that no 2 users, when browsing the same websites, would see or focus on the same things. Leading to a much more diverse range of experiences than our current experiences of the Internet, which are for the vast majority homogeneous and top-down defined.

In other ways, we felt that certain categories of websites, while neither advertisement, explicit or pernicious, represented parts that were unneeded or unwanted to us.

The modification program permanent-vacation constitutes an attempt to also shape the Web in this way.

The Stackoverflow website ${ }^{49}$, and the related Stack Exchange network of websites ${ }^{50}$, is an example of an information-driven, user-contributed platform where Internet and Web users can find information to specific topics of their choice. Stackoverflow is dedicated to computer science. Questions are tagged by specific interests (e.g. Haskell). Meanwhile Stack Exchange has various communities (e.g. Mathematics).

And, while the answers to questions themselves are relevant, and the related categories of questions often offers pertinent information, the "Hot Network Questions" features questions from the entire network (e.g. cooking) that are

\footnotetext{
49 https : //stackoverflow.com/

${ }^{50}$ https://stackexchange.com/
} 
off-topic.

\#\#\# Description

This extension removes the "Hot Network Questions" bug / feature

(depending on whether this is viewed from the dominant view of

the platform's, or the personal needs of the user), which

contains unwanted information ('digital/information

pollution'/'noise') unrelated to the original question/search.

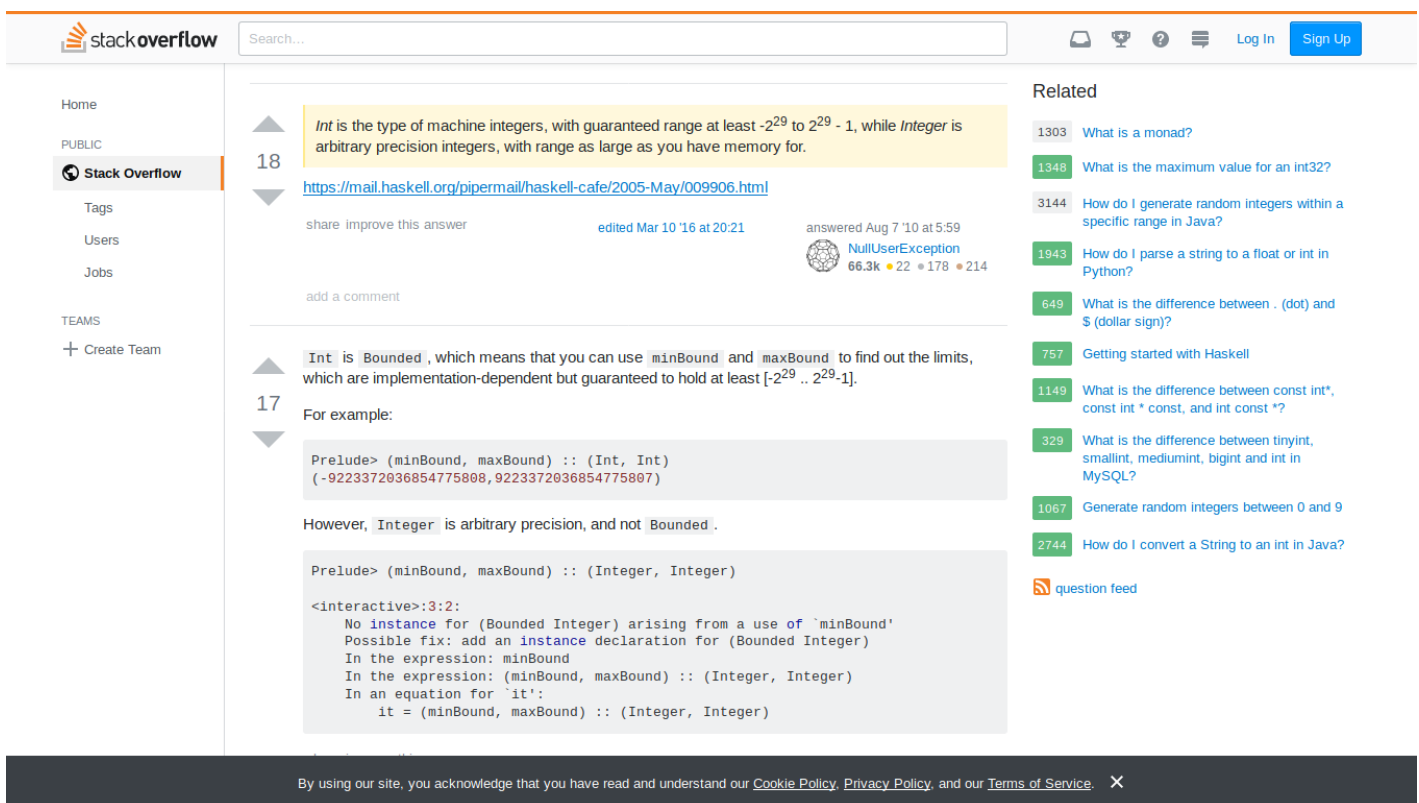

Figure 5: A user sees this while using the permanent-vacation program. Note the absence of further content below "Related"

\subsection{Obstacles}

Obstacles that stand in the way of either the creation or adoption of software can usually be subdivided into technical and sociological.

Currently, one of the obstacles that stands in front of the adoption of such a conception of the Web, is that such user-defined plug-ins need to be approved by a central authority, that will sign them, and perform additional operations on them for instance to make sure their contents are safe, and then return them to the user in a state such that will enable them to run them in any standard version of the browser (this is true of the Firefox browser).

Ways around this are currently the use of so-called "Nightly builds" of the Firefox browser, which are experimental versions of the browser. 
Add-ons need to be signed before they can be installed into release and beta versions of Firefox. This signing process takes place through addons.mozilla.org (AMO), whether you choose to distribute your add-on through AMO or to do it yourself.

Unsigned extensions can be installed in Developer Edition, Nightly, and ESR versions of Firefox, after toggling the xpinstall.signatures.required preference in about:config. ${ }^{51}$

The technological requirements, however extremely minimal as in the case of 'no-television' and 'permanent-vacation', (certainly represent nonetheless an obstacle. But, perhaps, these basic technological requirements will represent a basic skill in future generations, though this is matter of pure speculation, and, more pragmatically, of how education will be conceived of and shaped then - we say however nothing of whether this is something that should be wished for or expected or realized.

Further considerations, not so much obstacles, are that the web is a changing medium and these alterations (browser plug-ins) may have to be rewritten (at times) from time to time.

\subsection{A "User Owned" Internet}

It would be hard to write an article on the Web (a user-defined Web), without mentioning the Internet at more length, which constitutes, as we know both of them (currently), its backbone; and specifically the implications and dependencies of both on both.

And, so, we do not conclude this article by looking at alternatives for the Internet as well, which we do elsewhere, but by pointing to general trends.

While this article has mostly concentrated on the Web, it is important to note that movements and ideas neither emerge out of or exist on thin air. While the web is its layer of ideas, its infrastructure is and remains currently the Internet - that is for the great majority of people.

A free Internet is no guarantee for a free Web, but it is one of its conditions - one.

Other and current efforts have concentrated on the removal of structural dependencies that stand in the way of a democratic Internet (, and hence Web) ${ }^{52}$ - mainly the ownership of the Internet's infrastructures ${ }^{53}$ by few companies,

\footnotetext{
${ }^{51}$ https://developer.mozilla.org/en-US/docs/Mozilla/Add-ons/Distribution

${ }^{52}$ We use the term democratic here specifically in the sense of "of the many" / "of the people". We chose to discard the euphemism "a more democratic".

${ }^{53}$ What is also generally referred to as "critical Internet infrastructure", various links or lines including (currently) fiber optic cables, and services like DNS. Not least the submarine communications cables (sub-sea fiber optic cables) on which it (still) relies.
} 
characterized by high degree of concentration, these are among others the socalled "Tier 1" networks or providers : CenturyLink, Verizon, AT\&T, Tata Communications India, Liberty Global, etc.

Attempts of creating a "user owned" Internet, or Internet-like networks, have recently known several incarnations. ${ }^{54}$

\section{Programs that make us dream : Software and Systems modifications}

In this section, we more generally discuss programs that change the way we think of technology and society ${ }^{55}$.

In doing so, we develop a theory of them that emphasizes a value of programs not derived from or based on their - judged - technical merits, but their ability to extend the domain of what we think possible or permissible or desirable, our domain of possibilities in other words.

Indeed : With certain programs, it is not so much their technical intricacies - sometimes surprisingly small or limited - that impress us, or put us in awe, or makes them of importance to us, but the ways in which they shape or re-shape our understanding of the possible uses or meanings of technology, and their interactions with our societies.

They often do because they put upside down our conceptions of them, much of which we have inherited from dominant views, those of the manufacturers of hardware, makers of commercial software, large, enterprise corporations, but not least also from academic computer scientists, an ever-increasing portion of whom come from or have significant ties with these same groups, whose rules, disciplines, sets of thinkable and un-thinkable, permissible and non-permissible, acceptable and non-acceptable, they then bring home, in thought or unthought form, in the abstract form of grades, the classes they teach, the topics they select (or "prefer to confine themselves" to), the textbooks they pick, and in the way of the graduate students they select and do not select, colleagues ("coworkers") they promote and do no promote, and the others they publish and do not publish, and up to the things they themselves publish and encourage their students to publish, or discourage. ${ }^{56}$

Some books can have the power of a many Men, it has been observed, but the same can be said of programs; and, perhaps much more so. And, as for our times, as we can, for better or worse, only live in them, though some do their

\footnotetext{
${ }^{54}$ We discuss this topic in detail elsewhere, separately.

${ }^{55}$ By modifying either or both systems.

56 "Microsoft Research Cambridge, said the company had a long history of a two-way flow of researchers between itself and universities that was beneficial to all." (Sample 2017)
} 
best to ignore them, a keyboard can be much more powerful than a pen.

In the following developments, we look at two works in particular, keepgrabbing.py, and Kopimashin, that are representative of such programs. ${ }^{57}$

\subsection{Aaron Swartz' keepgrabbing.py}

An example of such a program is the 17-line keepgrabbing.py program by Aaron Swartz, reproduced here down below :

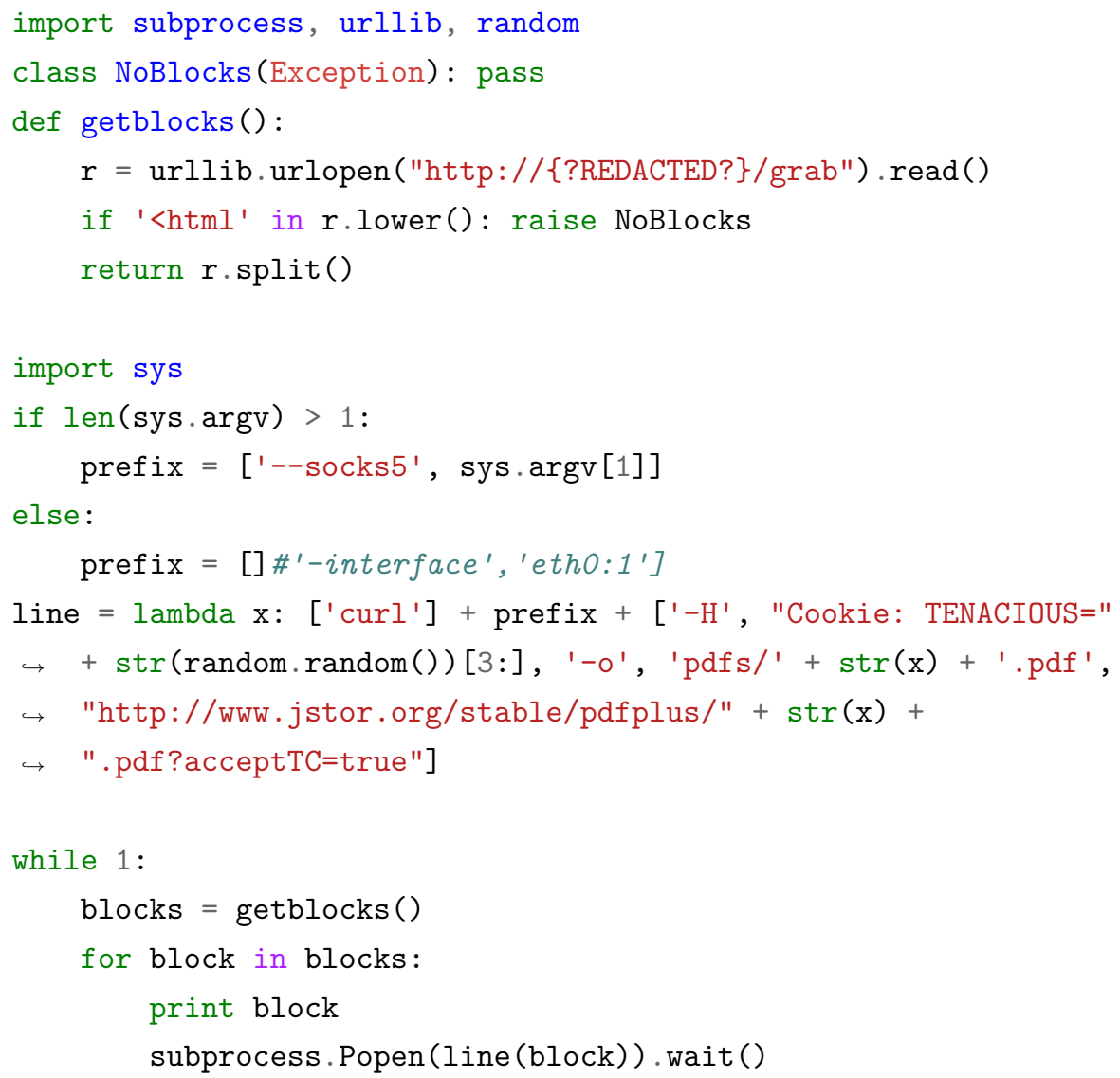

What puts us in awe, and fills us with wonder, whenever we encounter such programs are questions such as "What kind of upbringing made them possible? What kind of education? What collected life experiences made them ultimately thinkable?" Issues of technical prowess, here a lambda function ${ }^{58}$, lambda, or a

\footnotetext{
${ }^{57}$ We remind of the wider history of systems modifiers elsewhere. A separate forthcoming article.

${ }^{58}$ Lambda functions have the general form lambda $\mathrm{x}$ : do something with or to $\mathrm{x}$ e.g. lambda $\mathrm{x}: \quad \mathrm{x}+1$ such that e.g. lambda $1=2$. They go back to the lambda calculus of Alonzo Church, and various discussions of and improvements on it by others. In keepgrabbing.py $\mathrm{x}$ is
} 
reference to a redacted website of which we are left to wonder exactly what it did and how the /grab request was implemented, of which some technologists seemingly cannot get enough, to the exclusion of everything else, appear almost of secondary nature and importance when compared to these other questions.

But, such programs, do more than that : They extend the domain of our imagination, and ultimately possibilities : what was previously unthought, or thought impossible, suddenly becomes thinkable and possible.

A program such at this one would have represented no visible or particular challenge to someone like Aaron Swartz who had been programming since his teen years at least and who by his mid-twenties would have accumulated anywhere from 15 to 20 years of programming and computer science experience. ${ }^{59}$ But, what is true is that it took a special kind of courage to do it, and a special kind of heart and person to think it and want it ${ }^{60}$.

Again, it is not the complexity of the program, which, at under 20 lines of mostly self-contained code $^{61}$, can only be relatively small, that strikes, but what it means, and what can be made of it.

It effectively was able to download, by various tricks such as the insertion of headers with random values ${ }^{62}$ or MAC address changes ${ }^{63}$, massive amounts of

passed to other functions, namely the Python built-in function str which turns a value, here a number, into a string (text). This is explained in further detail in the appendix. There is no standard form for such functions as far as is known to us, and they can freely (re)written as e.g. $\backslash \mathrm{x}=\mathrm{x}+1$ (where the backslash is an approximation or simplification of the, lowercase, lambda symbol) or $\lambda \mathrm{x}=\mathrm{x}+1$, etc.

${ }^{59}$ The more surprising thing would have been for anyone to not have reached some kind of mastery, whatever the field, by then. In a post entitled "A Non-Programmer's Apology" particularly, he explains his commitment to learning his skills.

${ }^{60}$ We believe it was Evelyn Waugh who wrote, in Brideshead Revisited, that "intelligence without heart is sterile".

${ }^{61}$ It does not reference other functions that may be much more voluminous, other than standard library (subprocess, urllib, random and sys), here the "Python Standard Library", or other, third-party modules, libraries or programs (here, in addition, the utility curl is used). The only uncertainty is represented by the redacted website, which may have contained additional and possibly complex code, we do not know.

$62,-\mathrm{H}$ ', "Cookie: TENACIOUS $="+\operatorname{str}(\operatorname{random} . \operatorname{random}())[3:]$

${ }^{63}$ In official court documents, the following was noted : "To evade the MAC address block, Swartz "spoofed" the Acer laptop's computer's MAC address. A MAC address is usually assigned to a network interface card by the cards manufacturer, and therefore generally remains constant. But a user with the right knowledge can change the MAC address, an action referred to as "MAC address spoofing." Swartz spoofed the Acer laptop's MAC address by changing it from 00:23:5a:73:5f:fb to 00:23:5a:73:5f:fc (that is, the final 'b' became a 'c'). c. By re-registering the laptop, the laptop received a new IP address, which disassociated Swartz's Acer laptop from the IP addresses that JSTOR had blocked" (USA v. Swartz, p. 7). In the code, a reference to the network interface card can be found in the following line of code : \#'-interface', 'eth0:1' (note this is a comment, as per the \# symbol, which instructs the compiler to ignore whatever comes afterwards on that line, it is a form of documentation). The documents later mention : "Rather than let MIT assign his computer an IP address automatically, Swartz instead simply hard-wired into the network and assigned himself two IP addresses. He did so by entering a restricted network interface closet in the basement of MIT's Building 16, plugging the computer directly into the network (...) Swartz also hid the Acer laptop and a succession of external storage drives under a box in the closet, so that they would not arouse the suspicions of anyone who might enter the closet." (p. 8). 
articles from the JSTOR catalog of scholarly articles. But beyond this, it did more as we have already suggested.

And, though, this program had many antecedents in the "hacker" culture that had originated at MIT, first in the Tech Model Railroad Club, then around various Artificial Intelligence figures and projects, it also seemed to represent something else. ${ }^{64}$

It was not a "clever hack", and did not correspond to the old model of a, again, "clever hack", which is to say to prove or demonstrate to others technical prowess or bravado, it was not made for amusement or entertainment (Spacewar), or personal gain, whether financial or of mere service ${ }^{65}$, but seemed to aim at what could be described as the liberation of information for the public benefit; information that was for instance either in fact public, but hard to reach, out of whatever administrative or technical or legal constraints, or such information that was created as part of a publicly funded institution, but nonetheless remained out of reach to those, namely the people, who had funded it through taxes and various other means, etc..

Aaron Swartz' program represents a vision of a Web or an Internet that is about pure information and knowledge (transfer), devoid of graphical interfaces, uninterested in paywalls and the legally protected, but collectively harmful notion of a system of copyrighted, for-profit ${ }^{66}$, closed research publication model ${ }^{67}$.

The violations of the program were much more than the superficial downloading of scholarly articles, but the modifications it operated on our values (detailed in as many offenses listed in USA v. Swartz). Values that we hold on to despite better judgement, so the strange and peculiar notion of our times that scientific knowledge should or could be owned, instead of being accessible to the greatest number possible. But, how else is science expected to make progress? The more people work on a problem, and the more ressources they have access to, one influencing the other, the higher the chance of finding a solution. ${ }^{68}$

\footnotetext{
${ }^{64}$ Where the specific culture of "hackers" is concerned, and specifically its historical origins, both drastically different from common mainstream media usage and portrayal and perception, see Levy's Hackers: Heroes of the Computer Revolution. We talk about this further, and give a critical analysis, elsewhere, in a separate article.

${ }^{65} \mathrm{E}$.g. a printer is now endowed with functionalities it did not have before.

${ }^{66}$ In 2017, the publisher Elsevier has made revenues of 2.5 billion dollars (RELX Group 2017). It is hard to think of another market where participants and actors not only readily give away their labor, but pay to give it away in some cases, and attach great pride to these various acts.

${ }^{67}$ Currently, despite all the collective progress made, this is still the dominant model in research, particularly in the humanities and the social sciences (economics excluded perhaps), where researchers continue to fall over themselves to publish in their favorite journals, or any closed journal that will accept them, not out of lack of information or available alternatives, now plenty, but because - it must be said - they are either naive or slaves to and blinded by reputation, unable to draw the self-esteem they lack from the merits of their research alone, having to attach additional institutions to their name to make up for either or.

${ }^{68}$ This corresponds to a portion of how modern mathematics research is done, where not only the digital open-access platform arXiv exists which regularly is the place of publication
} 
Where Aaron Swartz goes, his biggest offense may have been to "ignore their laws and profane their holy culture" 69 . Such had always been the biggest crime of all. ${ }^{70}$

\subsection{Peter Sunde's Kopimashin}

Another creation that has fulfilled a similar role is Kopimashin ${ }^{71}$ by Peter Sunde.

A description for the Kopimashin has been provided as follows :

The Kopimashin creates an endless amount of copies of a specific audio track (gnarls barkley's crazy). The audio track is copied to /dev/null, a unix data pipe for avoiding permanent storage. The Kopimashins lcd display consists of three rows of information, the serial number of the mashin, amount of copies created and the dollar value it represents in losses for the record labels (Downtown Records / Warner Music), currently represented by USD1,25 per copied piece.

The goal of the kopimashin is to make the audio track the most copied in the world and while doing so bankrupting the record industry. ${ }^{72}$

In keepgrabbing, the traditional graphical interfaces of the Web were forgone to access information on the Web or Internet, but here even the data collected itself is forgone as soon as copied.

Kopimashin, the name we use here to describe the program contained within the Kopimashin, indeed transfers copyrighted material at a considerable rate, but as soon as a file is copied, it is instantaneously discarded (by being sent to the /dev/null location (data pipe), which in Unix systems, fulfills the function of erasing whatever is sent to it e.g. by using the redirect operator, >, like so someoperation > /dev/null, or by using a utility tool for copying, $\mathrm{cp}$ for example, like so cp filename /dev/null).

of major papers by leading mathematicians, but platforms such as wiki communities and communication tools such as chat programs are used to bring together researchers from all over the world to work on common, open problems. (This was for instance the case after the publication of Yitang Zhang's paper on prime number gaps, which led to a burst of activity in number theory, with groups forming around individuals who had already thought of or contributed to these problems e.g. Terrence Tao et al. used Skype to communicate.)

${ }^{69}$ One of Aaron Swartz' favorite TV series was Buffy, and we cite here with slight modifications from one of the so-called "Buffyverse" - a contraction of Buffy and universe - shows, called Angel (Season 2). He wrote about such things in various blog posts (that have now been published).

${ }^{70}$ The heroes of Ancient Greek tragedies saw a conflict between Earthly laws and the laws of the heart, and of Gods, burial rights in Antigone for instance, but still could not help but intervene, paying the high price. We remember them, protect their memory and learn from their experiences, cherish their existence that give us courage, and set an example.

${ }^{71}$ Whose use is demonstrated in the following video : https://vimeo.com/148955816. The graphic we present is extracted from this work.

72 "KH000 // Kopimashin". https://konsthack.se/portfolio/kh000-kopimashin/ 
From a "purely technical" standpoint, which is the preferred standpoint of mainstream computer scientists, such a program would make little sense.

However, in these sorts of programs, as we have already explained, it is not only the technical aspects that matter, but what they are able to accomplish.

In this case, such a program raises a host of questions on current copyright laws, and is able to do so most notably in a practical way : Does such an act constitute an infringement? The files, while technically copied, are instantly discarded and never used.

Kopimashin mocks copyright laws, and/but is able to do so from the internal perspective of technology, where the programmer of such programs is at an advantage, because they know and control and make the system, not least due to having created it.

This is as opposed to trying to modify copyright laws from the internal perspective of law, which is to say to fight laws with laws - a system in which programmers or any outsiders are and can only be at a disadvantage for the great majority of cases, and for the vast majority of them ${ }^{73} \cdot{ }^{74}$

It is also worthy to note that this machine (consisting of a popular embedded board, an LCD display, and the program itself) was showcased as part of an art exhibition $^{75}$.

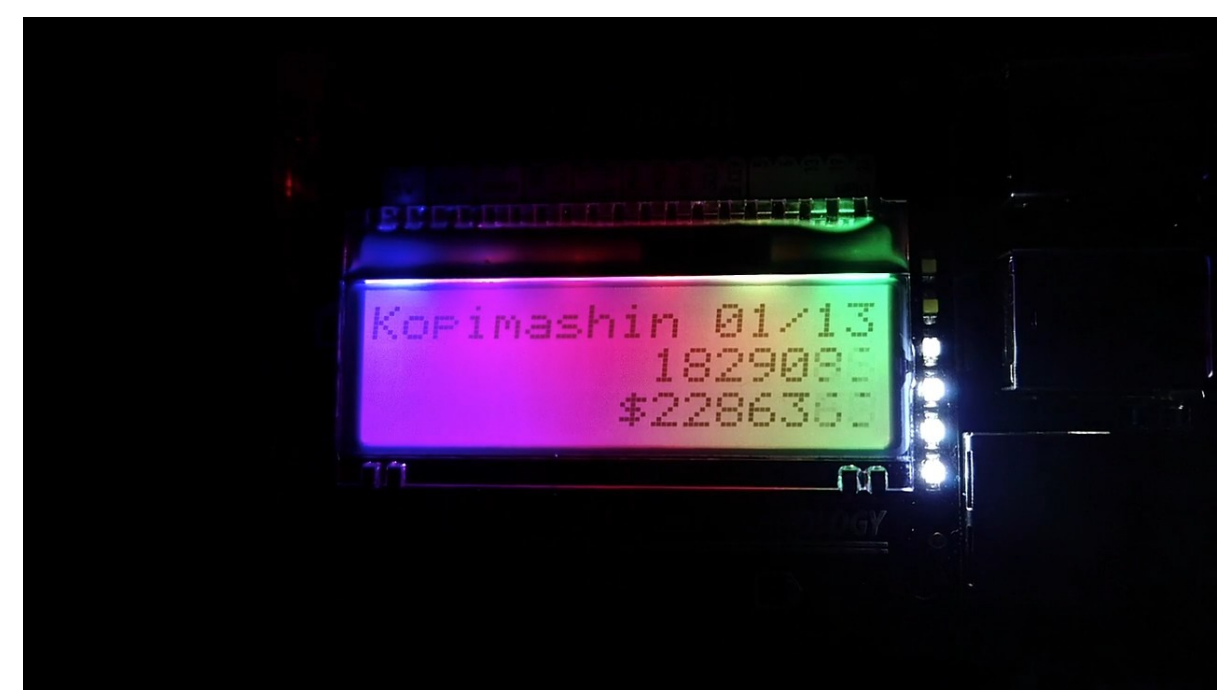

Figure 6: The Kopimashin shows potential damages of over $2 \mathrm{M} \$$ !

\footnotetext{
${ }^{73}$ Which, at the same time, does not mean they should completely forgo, or ignore this. Their expertise is valuable and needed there too.

${ }^{74}$ In a speech, Andrew Huang, better known as bunnie, recounting his experiences of hacking the Xbox, and expanding on them, mentioned that it was hard to fight a system from within the system's rules, as this could only put one at a disadvantage. One could or had to find ways to approach the system from within a set of rules it had itself not designed or implemented or made use of.

${ }^{75}$ Konsthack. https://konsthack.se/
} 
A pseudo-code for this program, which ignores the display, hence a simplified command-line version of it, could be written as follows :

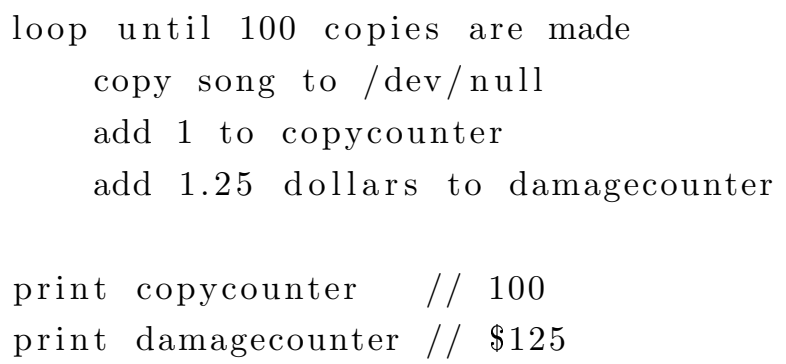

And, in a slightly more intricate variant that would begin to take account of a display, for visual, pedagogic or art purposes :

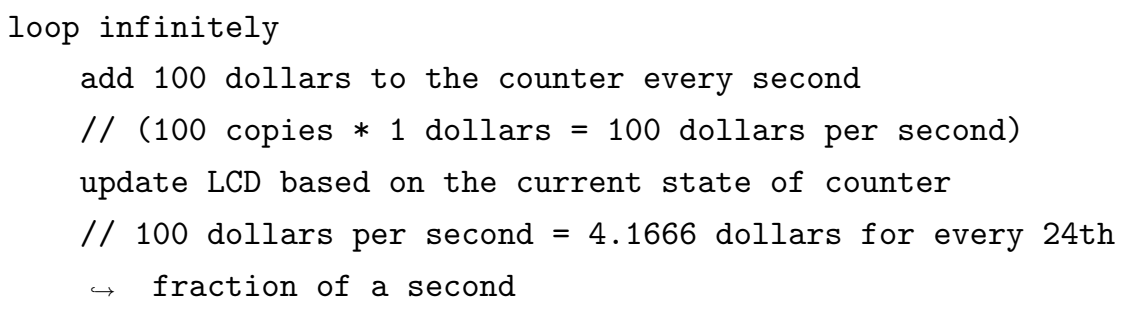

Lastly, such programs do not need to be laid out completely, or even be in working or final form. Steven Levy, in the documentary Hackers: Wizards of the Electronic Age, says "A hacker never finishes a program".

\section{Conclusion}

A "user-defined" Web was presented in opposition to dominant conceptions of a "user-centered" or "centric" Web; which we call falsely benevolent in so far as they are dependent on the good will of those who have control or an aboveaverage influence on the Internet and the Web, their nature, development and direction - and, worse, their collective agreement.

Opposite models, that are passed as progressive and in the interest of the user, such as "user-centric" or "user-centered" terminology, retain the quality of highly centralized systems where top-down decisions, here imposed from few companies, (leading) designers and the like on many users, and cannot reasonably thought to provide an alternative for a democratic Web.

We discussed systems modifications : "programs that make us dream" of a different society, and of different uses of technology - they do so often by modifying both. 
These programs make us dream of different, and better tomorrows. They give (offer us) glimpses into alternative futures, that could simultaneously be alternative presents. Programs. Programs. Programs. "Programs". Pro-grams. Grams. Pros, and cons. As many different visions of the ways we may organize our societies - open or closed, by few or by many - and design our many systems, of which not only electronic or software. Knowledge, politics, etc.

Good books should not have the effect of making us think of lunch. They should make us weary, and out of breath, and out of sight. Nauseous and out of balance, no maps can seemingly be found. For what had been held for granted, is not only false, but can be modified.

Not, just in words - the words and ways of Goldsmith-style academics, bowl cuts and affected false-queer styles and all, whose lives are often nothing to write about, either because they are mundane, or of no standard to follow, or both. So much different from their models'. Foucault had lived a thousands of them, and each one with much courage : he wrote perfect-shaped books, dazzling like black diamonds, impressive even by the standards of Medieval copists, but it was the same person who went out on the streets for those in prisons, the same who had been in a relationship with a crossdressing prostitute, the same who had been in a psychiatric hospital when he was younger, the same who planned on becoming a journalist because he had had enough of being what he was, and what they could only dream to be, and would never be even if they had a thousands lives, the same who, when he died early, left many confused, for, just for a short while, it seemed like they had had a glimpse of tomorrow;

But, in real acts. Programs are political acts. 


\section{Bibliography}

Berners-Lee, Tim. 1999. Weaving the Web: The Original Design and Ultimate Destiny of the World Wide Web by Its Inventor. San Francisco, CA : Harper San Francisco.

—. 2018. "The web is under threat. Join us and fight for it."

https://webfoundation.org/2018/03/web-birthday-29/

—. 2019. "30 years on, what's next \#ForTheWeb?"

https://webfoundation.org/2019/03/web-birthday-30/

FitzGerald, Drew and Wakabayashi, Daisuke. 2014. "Apple Quietly Builds New Networks". The Wall Street Journal, 03/02.

https://www.wsj.com/articles/apple-quietly-builds-new-networks-1391474149

FitzGerald, Drew. 2014. "Netflix's Share of Internet Traffic Grows". The Wall Street Journal, 14/05.

https://www.wsj.com/articles/netflixs-share-of-internet-traffic-grows1400079287

Hafner, Katie and Lyon, Matthew. 1998. Where Wizards Stay Up Late: The Origins of the Internet. New York, NY : Touchstone.

Huang, Andrew. 2018. "Making \& Breaking Hardware: Origins of the Hacker Ethos". https : //www . youtube. com/watch?v=HwkbbVoQOxw

Kurose, James and Ross, Keith. 2017. Computer Networking: A Top-Down Approach. Harlow : Pearson Education.

Marx, Karl. The German Ideology, in particular I. Feuerbach.

Tanenbaum, Andrew. 2003. Computer Networks. Upper Saddle River, NJ : Pearson Education.

RELX Group. 2017. "Annual Reports and Financial Statements 2017". https://www.relx.com/ /media/Files/R/RELX-Group/documents/reports/ annual-reports/relx2017-annual-report.pdf

Sample, Ian. 2017. "Big tech firms' AI hiring frenzy leads to brain drain at UK universities". The Guardian, 02/11.

Swartz, Aaron. http://www.aaronsw.com/weblog/

"UNITED STATES OF AMERICA v. AARON SWARTZ". https://www.docketalarm.com/cases/Massachusetts_District_Court/1--11cr-10260/USA_v._Swartz/53/ 
$\underline{\text { keepgrabbing.py }}$

Collective. "Dissecting the script Aaron Swartz used to download several thousand articles from Jstor's archive".

https://stackoverflow. com/questions/49711153/dissecting-the-scriptaaron-swartz-used-to-download-several-thousand-articles-fr

"keepgrabbing.py". https://github.com/sprky0/jstor

"UNITED STATES OF AMERICA v. AARON SWARTZ".

https://www.docketalarm.com/cases/Massachusetts_District_Court/1--11cr-10260/USA_v._Swartz/53/

"The Python Standard Library". https://docs.python.org/3/library/

ProPublica. Nonprofit Explorer. ITHAKA HARBORS INC. https://projects . propublica.org/nonprofits/organizations/133857105

Kopimashin

"KH000//Kopimashin". https://vimeo.com/148955816

"KH000 // Kopimashin". https://konsthack.se/portfolio/kh000-kopimashin/

Konsthack. https://konsthack.se/ 


\section{Acknowledgements}

Dedicated to J., with, much, much love. 


\title{
Appendix
}

\author{
- no-television \\ - permanent-vacation \\ - Website YouTube on 15/12/2005 (19:40:08) (official launch day). \\ - Analysis, documentation of keepgrabbing.py, hand calculations
}


no-television.js

document.body.style.visibility = "hidden"; // optional

window.location.href $=$

$\hookrightarrow$ "https://www. youtube.com/results?search_query=\%2A"; // "*"

// could be set to search for a random word from a dictionary

$\hookrightarrow \quad$ (list)

/* working solution

var elem = document.getElementById("page-manager");

elem.remove (); 


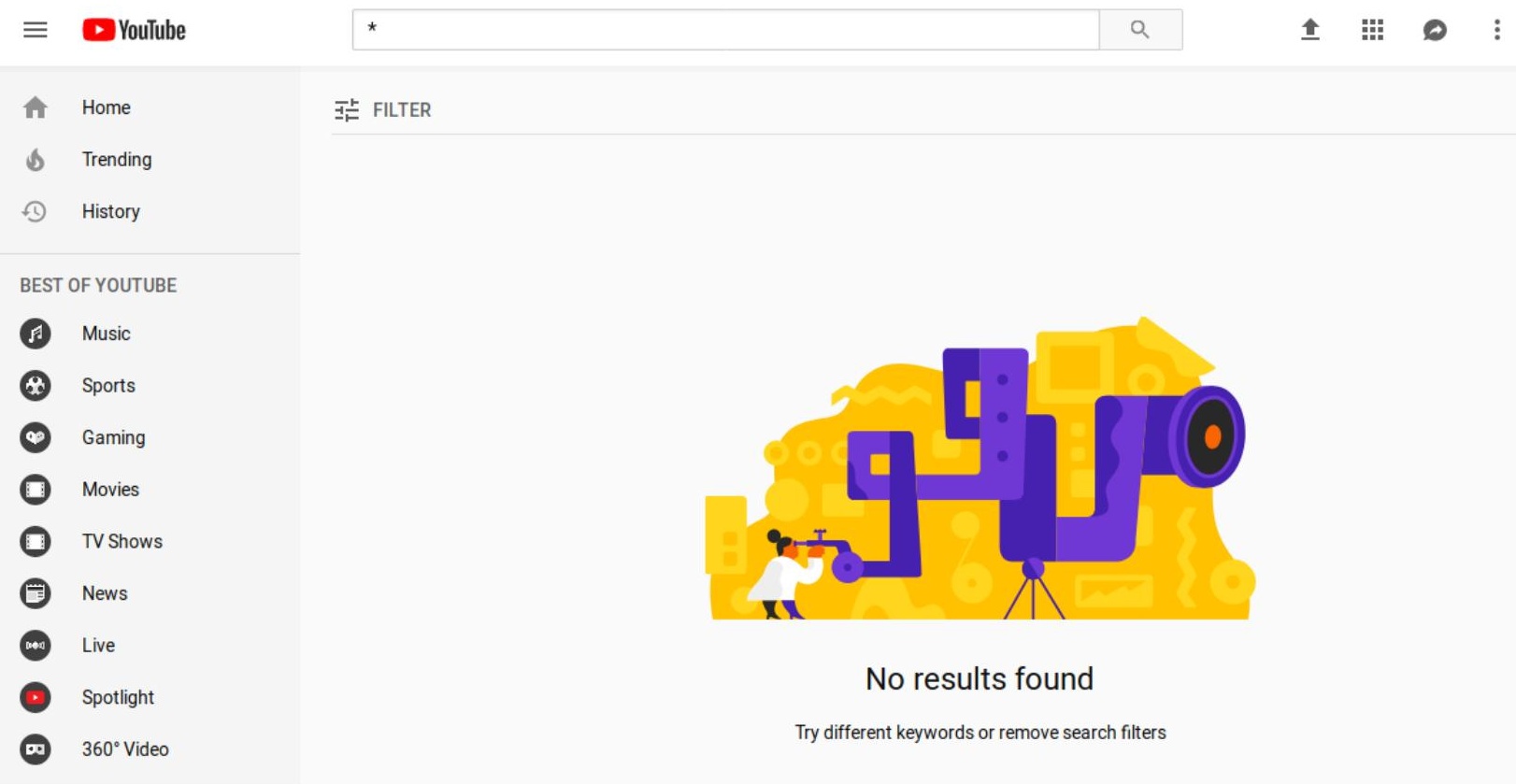

Figure 7: no-television, home page view (current version). 
permanent-vacation.js

var elem = document.getElementById("hot-network-questions");

elem.remove(); 


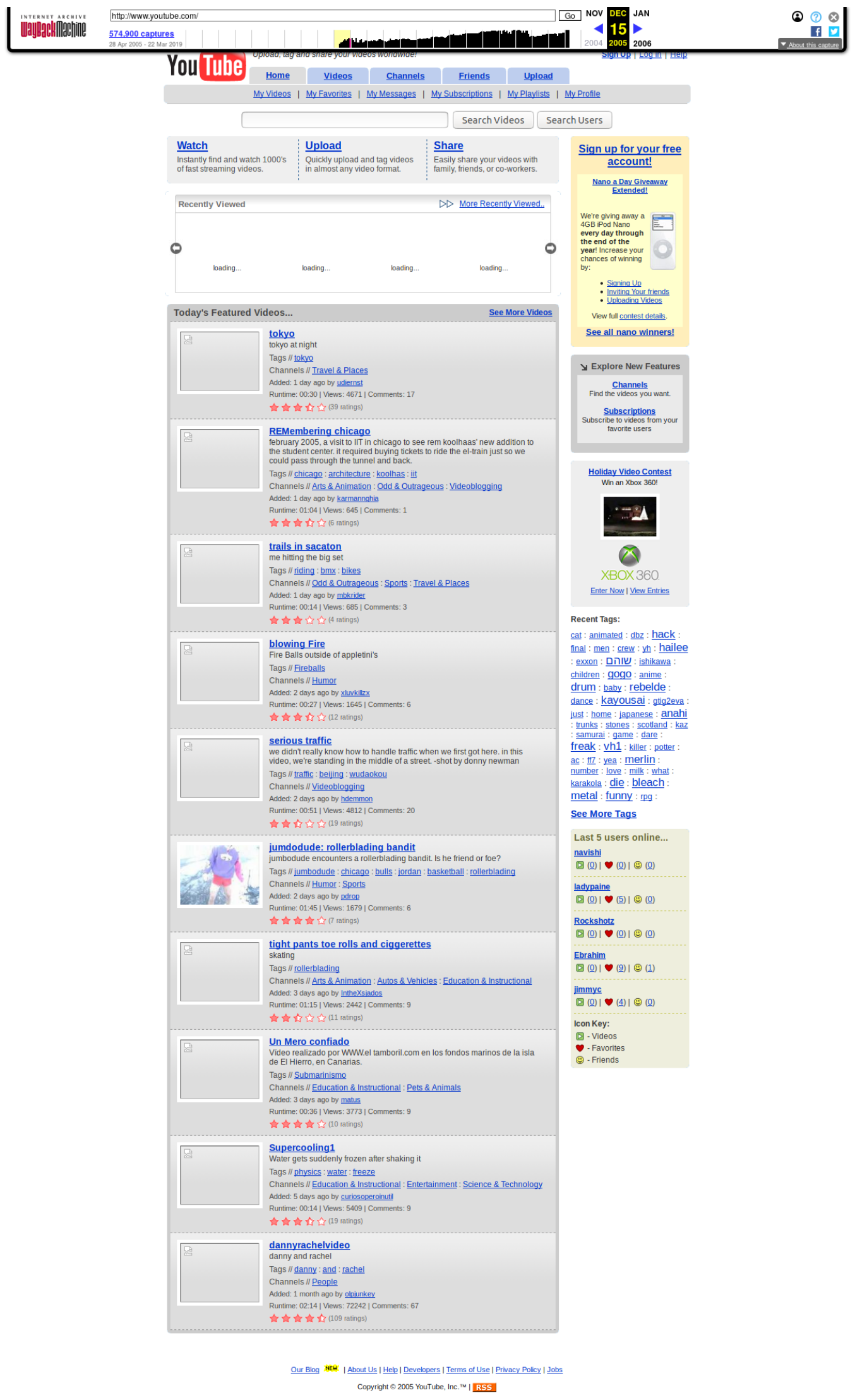

Figure 8: Website YouTube on 15/12/2005 (19:40:08) (official launch day). 
Analysis, documentation of keepgrabbing.py, hand calculations

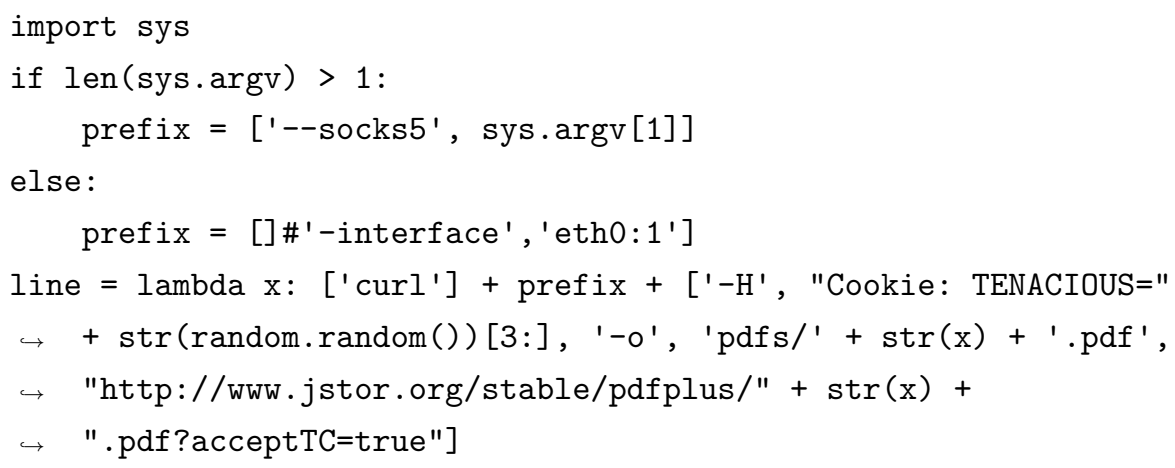

\$ python

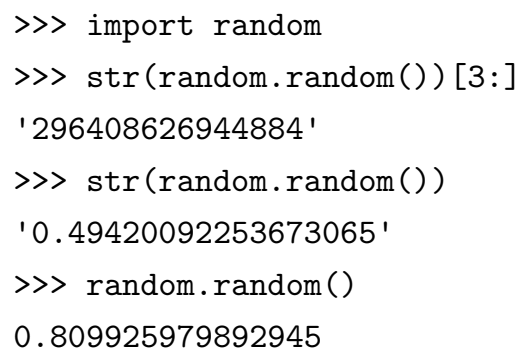


NAME

curl - transfer a URL

SYNOPSIS

curl [options] [URL...]

DESCRIPTION

curl is a tool to transfer data from or to a server, using one of the supported protocols (DICT, FILE, FTP, FTPS, GOPHER, HTTP, HTTPS, IMAP, IMAPS, LDAP, LDAPS, POP3, POP3S, RTMP, RTSP, ...).

\section{OPTIONS}

Options start with one or two dashes. Many of the options require an additional value next to them.

-H, --header <header/@file>

(HTTP) Extra header to include in the request when sending HTTP to a server. You may specify any number of extra headers. Note that if you should add a custom header that has the same name as one of the internal ones curl would use, your externally set header will be used instead of the internal one. This allows you to make even trickier stuff than curl would normally do. You should not replace internally set headers without knowing per fectly well what you're doing. Remove an internal header by giv ing a replacement without content on the right side of the colon, as in: -H "Host:". If you send the custom header with novalue then its header must be terminated with a semicolon, such as -H "X-Custom-Header;" to send "X-Custom-Header:".

curl will make sure that each header you add/replace is sent with the proper end-of-line marker, you should thus not add that as a part of the header content: do not add newlines or carriage returns, they will only mess things up for you. 
-o, --output <file>

Write output to $\langle$ file $\rangle$ instead of stdout. If you are using \{\} or

[] to fetch multiple documents, you can use '\#' followed by a

number in the <file specifier. That variable will be replaced

with the current string for the URL being fetched. Like in:

curl http://\{one,two\}.example.com -o "file_\#1.txt"

--socks5 <host [:port]>

Use the specified SOCKS5 proxy - but resolve the host name locally. If the port number is not specified, it is assumed at port 1080 . 